\title{
4 Dos sistemas, dos campos y, finalmente, dos lógicas
}

En la bibliografía especializada suelen aparecer, hasta el momento sin mayores análisis, menciones a los órdenes diferenciados que constituyen la literatura nacional y la literatura mundial. Por otro lado, he introducido en los apartados anteriores el término lógica para referirme a los principios generales que regulan el funcionamiento de los campos literarios y la producción de literatura. En las próximas páginas me interesa profundizar en tales aspectos para tratar de articular un modelo que permita explicar mejor tanto la circulación como la no circulación de productos literarios.

Según ya se expuso, Damrosch sostiene que aquellos textos que responden a un conocimiento cultural demasiado específico no reúnen condiciones para trascender las fronteras de la literatura nacional y, en consecuencia, no pueden ser acogidos jamás en el corpus de la literatura mundial. Por esta razón, de un modo similar a como lo propone Moretti, el estudio de esta última sería competencia de investigadores adiestrados en los parámetros de circulación y recepción a escala mundial. Si para Moretti el método de close reading queda reservado para el investigador de las literaturas nacionales y el distant reading, para el de la mundial, para Damrosch esta "división internacional del trabajo" (Moraña, "Post-scriptum...” 327) se distribuiría, aunque con algunas reservas que lo distinguen de Moretti, entre "especialistas" y "generalistas": "Intimately aware of a work's life at home, the specialist is not always in the best position to assess the dramatically different terms on which it may engage with a distant culture. Looking at such new context, the generalist will find that much of the specialist's information about the work's origins is no longer relevant and not only can but should be set aside" (287). Los conocimientos que permiten abordar un texto en su performance a nivel nacional no serían, así, adecuados para hacerlo en su eventual circulación internacional. Esto se debe a que las pautas de producción, puesta en circulación y recepción serían sensiblemente diferentes en ambos niveles. El rendimiento que se le pide a un texto a nivel internacional no es equiparable, ni susceptible de ser evaluado, desde una perspectiva arraigada a los parámetros nacionales o locales. Este desdoblamiento de los escenarios de acción de la literatura en dos órdenes claramente diferenciados va a aparecer con frecuencia en la bibliografía especializada sin que se lo someta a exámenes mayores. También Mads Rosendahl Thomsen da cuenta de este desdoblamiento; anota que "The importance of formal and thematic properties to international canonization has been underestimated and understudied, especially as national canonization has a different logic and different values than 
international canonization" (3). Se trata, pues, no solo de que los modos de lectura necesarios para abordar la literatura nacional y la mundial deben ser diferentes, sino también de que los atributos formales y temáticos que deben reunir los textos para insertarse exitosamente en una $u$ otra estructura de circulación no son los mismos o no son percibidos de la misma manera.

Sin duda, el repertorio de valores culturales que van a favorecer o no la recepción de un texto en un nivel y en el otro no son en modo alguno equiparables. Tampoco las motivaciones históricas favorables a algunos temas y refractarias a otros. Por esta razón, difícilmente se podría pensar que las lecturas y modos de abordajes correspondientes a cada nivel se pueden fundar en las mismas premisas. O que los textos podrían circular bajo las mismas condiciones en ambos. Pero los dos niveles son algo más que espacios de recepción de literatura organizados a partir de pautas y tradiciones culturales diferenciadas. También funcionan como mercados con sus complejidades, desafíos y actores- en torno a los cuales, particularmente en el contexto de la actual fase de la globalización, toman forman campos literarios con "lógicas", como anota Rosendahl Thomsen, intrínsecas y desiguales.

También en la misma línea, pero desde una óptica centrada en la industria editorial, Escalante Gonzalbo argumenta que "Hay autores que con independencia de su calidad se consideran 'universales' -y tienen distribución internacional-y los hay que se clasifican como provincianos, de interés local nada más. Son dos procesos distintos de producción y dos canales de distribución, y los libros se sitúan en uno o en otro: en el que se queda en el mercado local o el que llega a otros mercados" (282). Estamos, por lo tanto y en breve, ante dos sistemas productivos, sin duda con conexiones y tránsitos ineludibles, pero, al mismo tiempo, regulados por principios y concepciones de valor que los delimitan claramente. Por eso, el sistema de la literatura mundial requiere convenciones de lectura que no resultan adecuadas para el sistema nacional o local, y viceversa. Cada uno de estos dos sistemas, para acentuar el uso de un concepto más genérico y apropiado, que -repito- configuran a la vez un campo, una estructura productiva y un mercado, poseen, respectivamente, una lógica, esto es, un repertorio de reglas que pautan su funcionamiento. De acuerdo con John Thompson, "each field of publishing has a distinctive dynamic - what I call 'the logic of the field'. The logic of a publishing field is a set of factors that determine the conditions under which individual agents and organizations can participate in the field - that is, the conditions under which they can play the game (and play it successfully)" (11). La lógica que gobierna la producción y puesta en circulación de literatura en el campo internacional y determina bajo qué condiciones es posible participar en él resulta, en tanto intrínseca a este, 
diferente de la que pauta el funcionamiento de los campos locales. No solo porque las referencias culturales y los horizontes de expectativas no son compartidos, sino también porque las estructuras productivas y las condiciones materiales de producción -en particular si se dirige la atención a los campos periféricos- son sensiblemente disímiles.

En lo que sigue me interesa profundizar en algunos de los factores que hacen a estas dos lógicas diferenciadas. Antes de ello, sin embargo, propongo retomar los postulados de Boaventura de Sousa Santos ya avanzados en páginas anteriores. Si por nueva literatura mundial se entiende la que, actualmente, pone en circulación la industria editorial internacional con fines, en primer término, comerciales, las literaturas locales -en ocasiones, pero no siempre, admitidas como nacionales- son aquellas que suelen no recibir atención internacional, que no ingresan en circulación más allá de su contexto de producción original y las que, en consecuencia, son invisibilizadas como productos sin valor "universal". Se trata de la literatura escrita por los autores "provincianos" que menciona Escalante Gonzalbo y que, para Damrosch, reclama conocimientos culturales demasiado específicos. Nos referimos, por lo tanto, al corpus literario donde están contenidas y se expresan particularidades subalternas del mundo, es decir, las literaturas locales o que "dependen de contextos". Y no es necesario pensar en expresiones indígenas o de grupos minoritarios para hacerse una idea de este corpus, sino, antes y fundamentalmente, en la denominada publicación independiente y/o autogestionada con baja proyección de ventas y escasa cobertura territorial. Aplicar la sociología de las ausencias a la literatura mundial consiste, por lo tanto, en revelar esa zona de la producción de literatura a la que tanto el campo como el mercado de las particularidades dominantes a escala global suele no depararle atención por considerarla, precisamente, "provinciana” (cfr. Locane "Por una sociología...").

Dicho esto, avancemos con los atributos que distinguen los sistemas de la literatura mundial y de las literaturas locales, respectivamente.

En su estudio sobre la evolución de la industria editorial, John Thompson identifica al menos tres grandes factores como los responsables de vehiculizar el gran cambio de paradigma que, según sostiene, comienza a tomar forma en los años 60 y termina por concretarse en los 90 (102): “three key developments - the growth of the retail chains, the rise of literary agents and the consolidation of publishing houses under the umbrellas of large corporations - have shaped the evolution of trade publishing in the English-speaking world since the 1960s. These three developments have created a field which has a certain structure and dynamic or 'logic'” (291). Las corporaciones multimedios, los agentes y las cadenas de ventas conforman, así, los pilares sobre los que se asienta la (nueva) lógica del campo. Si bien Thompson se esfuerza por dejar en claro que 
su análisis se concentra en el mercado anglófono, también es cierto que ese modelo de gestión se propaga más allá de sus fronteras para penetrar campos específicos y dominar, sobre todo, el de la esfera internacional (cfr. Casanova cap. 5), la cual va a aparecer representada, ejemplarmente, por la Feria del Libro de Frankfurt. La lógica del mercado anglófono se habría convertido, así, en paradigma internacional e ideal a alcanzar por los campos locales. El mismo Thompson aprueba esta afirmación cuando escribe que

The fact that the Anglo-American publishing industry is the dominant industry in the international arena of trade publishing today is not accidental: it is rooted in a long historical process, stretching back to the nineteenth century and before, which established the English language as the de facto global language and gave Anglo-American publishers an enormous competitive advantage vis-à-vis their counterparts in other languages, who found themselves operating in much smaller and more restricted fields. (13)

La circulación internacional de literatura, por lo tanto, estaría reproduciendo un modelo que viene siendo ejercitado en el campo más específico del mundo anglófono. Sin embargo, habría que considerar algunas complejidades propias del escenario internacional, puesto que el cruce de fronteras por parte de un texto suele demandar la intervención de actores y un incremento de costos ausentes en el escenario más estrictamente local. La cadena de agregado de valor, cuando se realiza el salto al dominio internacional, se complejiza con la intervención de scouts, agentes, traductores, mayores costos de logística y distribución y presencia en eventos internacionales.

La mediación de agentes literarios que determinan los valores de los derechos de publicación y/o traducción más sus propios honorarios es un mecanismo ineludible en los tráficos internacionales. Introducen, sin disimularlo, una racionalidad economicista en el proceso que resulta necesaria para sostener los canales de circulación activos a largo plazo. Anne Marie Vallat, una agente con una importante trayectoria en el mundo hispánico, por ejemplo, afirma: “Al decidir si represento a un autor, influye el factor comercial. Esto es una empresa, no una ONG” (Paniagua). Un libro, a este nivel, -se comprende- debe asegurar ventas porque, de otro modo, todos los mecanismos de la mediación se verían a mediano plazo damnificados y, finalmente, renunciarían a sus funciones. El valor que la mediación de los agentes agrega al producto debe ser, a su vez, recuperado por medio de una posterior política de ventas, promoción y distribución eficiente.

Los costos de traducción, el honorario que un traductor debe percibir por su trabajo, es otro factor que en el escenario internacional se convierte en gravitante y que, finalmente, impacta en la lógica con la que los textos se seleccionan y entran en circulación. Ese costo, que suele ser adelantado por las editoriales, 
también va a tener que ser recuperado en la fase de ventas, lo que implica que tirajes, y mucho menos ventas, por debajo de determinado rango no pueden ser consideradas porque estarían atentando contra la dinámica estructural. Por otra parte, la comercialización de derechos de traducción adquiere la forma de un negocio per se, esto es, que el objetivo de algunos agentes y otros representantes no va a ser tanto conseguir que un libro produzca ventas y entre en efectiva circulación, sino, antes, vender la mayor cantidad posible de derechos de traducción. De acá se infiere que los textos que por un bajo nivel de exigencia formal o escasez de marcas socio o etnolingüísticas favorezcan el proceso de traducción van a recibir mayor atención que los que presenten resistencias.

Los libros que han pasado por estos procesos, que han acumulado valor agregado, deben, a su vez, llegar a la mayor cantidad posible de lectores, no necesariamente para producir un excedente exorbitante, pero sí al menos el retorno correspondiente al dinero invertido. En esa instancia es donde las bocas de ventas, las distribuidoras y los medios de difusión, como suplementos culturales, hacen su contribución al proceso para asegurar que el producto acabado alcance un público los más amplio posible. Como el valor económico del libro suele ser fijo, ${ }^{1}$ como no siempre está sometido a la ley de la libre competencia, el incremento de valor generado durante el proceso debe prorratearse en una inflación de oferta. Cuanto mayor ha sido el costo de producción, mayor debe ser la cantidad de ejemplares efectivamente distribuida y exitosamente vendida.

Este complejo proceso tiene -y de esto se trata finalmente- un impacto en la literatura mundial si la entendemos como aquella zona de la literatura que entra, fundamentalmente en traducción, en circulación internacional, pues los únicos capaces de cubrir los costos de gestión y mantenimiento de la infraestructura material y simbólica necesaria serían, en principio, los grandes grupos multimedios. $^{2}$ De acá se sigue que la literatura mundial hoy en día estaría controlada,

1 Leyes que fijan el precio del libro existen, fundamentalmente, en los países de Europa central, Francia, Alemania, Italia, España, Portugal y Holanda, no así en el mundo anglosajón. En América Latina, a su vez, la política regulatoria se encuentra, aunque en diferentes grados según los países, bastante extendida.

2 Advierte bien Malena Botto sobre los riesgos e ilusiones implicados en ese proceso. Cita a Bernardo Jaramillo, director, en el 2003, del Centro Nacional para la Industria Gráfica de Colombia, cuando este anota que "solo empresas estructuralmente fuertes pueden entrar a competir de lleno en el mercado internacional de derechos de autor, ingresar al mundo de las traducciones y expandir sus capacidades de mercadeo a toda le región”, y, a continuación, agrega que "lo que Jaramillo califica como 'reto y oportunidad' es en muchos casos, para las editoriales con menores recursos, una imposibilidad" (222). Se trata de que lo que algunos gestores culturales como Jaramillo ven como un aliento a la profesionalización de las pequeñas 
principalmente, por estos grupos y por los agentes. Mientras que los primeros disponen del capital requerido para mantener los canales en funcionamiento, los segundos son los encargados de proveer materias primas y contenidos. Pero junto con los factores materiales que condicionan la dinámica de la literatura a nivel mundial, habría que considerar que estos mediadores, al intervenir, imponen una lógica que resuelve la tensión entre la zona autónoma y la heterónoma del campo decididamente en favor de la segunda. Esto significa que los editores “culturales” que en los años 60 promovían la circulación internacional de Juan Carlos Onetti o Manuel Puig con el argumento de que sus escrituras poseían "valor literario" hoy en día se han visto a ceder no solo protagonismo concreto, en el mejor de los casos a quedar postergados en las tomas de decisiones, sino también protagonismo ideológico. Así, la industria editorial internacional, organizada en torno a la perspectiva de agentes y gerentes, gana libertad para guiar la configuración del futuro canon literario internacional bajo criterios de ventas. Esto implica que para la academia y la crítica internacionales -y acá el término “campo" de Bourdieu, en el uso que todavía le quiero dar, adquiere su significado más pleno- estaría subordinada y condicionada por los criterios de selección y puesta en circulación establecidos por la industria. El campo literario internacional aparecería, así, dominado ideológicamente por la zona heterónoma del campo: un buen libro es, finalmente, el que se vende. Y esta crucial reconceptualización del valor literario llegaría a tener una influencia, incluso, en los estudios académicos: los libros que van a resultar privilegiados en la investigación internacional van a ser, precisamente, aquellos que fueron seleccionados por la industria editorial. Así, para el caso de la literatura latinoamericana de reciente puesta en circulación internacional por mediación de agentes y editoriales con base de gestión en España, Pablo Sánchez escribe que "no sabemos si la 'herejía' literaria que ahora promueven las editoriales españolas (una red informal compuesta por Volpi, Padilla, Paz Soldán, Roncagliolo, Iwasaki, Fresán, Thays y tantos otros) quedará convertida finalmente en nueva ortodoxia dentro de unos años, pero la estrategia está en marcha y algunas consecuencias ya son comprobables” (“Un debate...” 25). Este corpus emergente de literatura latinoamericana para el mundo suele ser seleccionada y organizada en España, y más exactamente en las oficinas de agentes y directivos de Planeta y Random House/Alfaguara, de allí es transferido al mundo como escritura latinoamericana o en casos literatura a secas, lo cierto es que la

editoriales locales, en realidad no estaría evaluando correctamente las enormes diferencias que, en lo que refiere a control de recursos y posibilidades, distinguen a estas últimas de un grupo transnacional. 
academia y la crítica internacional suelen aceptar, sin interrogarla mayormente, esa selección. ${ }^{3}$

Como consecuencia formal de esta lógica, a su vez, habría que señalar una tendencia al “contenidismo". La nueva literatura mundial sería ante todo informativa porque responde a coyunturas históricas que despiertan el interés del púbico internacional por determinados temas: la "transición" en Cuba, la "situación política” en Venezuela, la "violencia” en Brasil, o la "paz" en Colombia pueden ejercer como datos extraliterarios que alimentan la demanda internacional de literaturas extranjeras. Son temas, además, regularmente abordados desde ópticas que coinciden con el ideario de la democracia liberal occidental y que, por consiguiente, no ponen en entredicho el lugar del lector en el mundo. Al contrario, estás literaturas tienden a constatar la percepción del mundo del lector occidental promedio. La literatura mundial, en este sentido, es aquella que, producida por la cadena de mediación donde agentes y gerentes son protagonistas, constata el orden geopolítico mundial y la distribución de competencias establecidos. La literatura mundial, esta, no conduce a una redefinición del yo constitutivo del sujeto consumidor sino a su confirmación como tal y a su representación etnocéntrica del mundo.

Esto también supone un relativo bajo riesgo formal en los textos de la literatura mundial. Puesto que el público al que se apunta debe ser, por razones de rendimiento económico, lo más amplio posible, y puesto que esa literatura no debe poner en cuestión el lugar en el mundo de ese lector para no afectar su deseo de consumo, todo riesgo formal excesivo resulta contraproducente para la literatura a este nivel. También porque implica desafíos para la traducción que en lo posible deben ser evitados. De acá -vale aclarar- no necesariamente habría que deducir que en la nueva literatura mundial no exista la experimentación formal, sino, más bien, que esta no se encuentra entre las prioridades de quienes toman las decisiones destinadas a seleccionarla y jerarquizarla, y que, si efectivamente es constatable, esto ocurre a pesar de los mecanismos de mediación y las instancias de filtrado.

Al otro lado, sin embargo, invisibilizada por las teorías hegemónicas de la literatura mundial, se registra una vasta producción que ya he sugerido caracterizar como literaturas locales y que su develamiento sería competencia primordial de una sociología literaria de las ausencias. Esta producción se encuentra sometida a otra lógica y corresponde, por lo tanto, asignarla a otro campo, a otra

3 Juan Gabriel Vásquez, valga el caso como ejemplo, recibió el premio Alfaguara en 2011; el 1 y 2 de octubre de 2015 se realizó el "Colloque international Juan Gabriel Vásquez: une archéologie du passé récent” en la Universidad Paul-Valéry de Montpellier. Andrés Neuman, por su parte, lo obtuvo en 2009; mientras que el 21 y 22 de mayo de 2012 tuvo lugar el "Coloquio Internacional Andrés Neuman” en la Universidad de Neuchâtel. 
dinámica productiva y a otro mercado. Es cierto que los límites entre el sistema mundial y los locales poseen ciertas porosidades y transiciones. ${ }^{4}$ También que la lógica del campo internacional llega a penetrar e influir en los segundos. Pero hay indicadores materiales que permiten establecer una diferenciación general, particularmente si se observan las dinámicas productivas de América Latina. Esto significa que no solo los horizontes de expectativas, los valores culturales y los conocimientos requeridos para el abordaje de literatura en los escenarios locales difieren de lo que sucede en el internacional, sino que la estructura productiva también posee rasgos propios.

Vamos a caracterizar a la estructura productiva mundial, la que requiere necesariamente la participación de actores altamente especializados, como "profesional". ${ }^{5}$ En el campo internacional los profesionales de la literatura regulan su funcionamiento e introducen mecanismos para tornarlo económicamente eficiente. Una estructura de edición profesional distribuye también beneficios económicos de manera que todos los actores que participan en la cadena de agregado de valor reciben su correspondiente compensación económica. ${ }^{6}$ Las

\footnotetext{
4 Para un estudio, por ejemplo, de cómo las editoriales independientes latinoamericanas operan como plataforma para un posterior lanzamiento de escritores al circuito internacional, véase el artículo de Ana Gallego Cuiñas "Las narrativas del siglo XXI en el Cono Sur. Estéticas alternativas, mediadores independientes".
}

5 Mario Vargas Llosa observa que Carmen Balcells habría sido la principal responsable, precisamente, de la profesionalización de los editores españoles que promovieron la literatura latinoamericana del boom. Al respecto, escribe: "les había hecho un inmenso servicio [a los editores], obligándolos a salir de las cavernas y asumir la actualidad. Porque si se conceden buenos anticipos y se aceptan tiempos límites para la explotación de unos derechos, los editores no tienen otro camino que promover bien los libros, y aguzar el ingenio para llegar a los lectores, y extender sus redes de distribución y conquistar nuevos mercados. Todo eso ha sucedido en la industria editorial de nuestra lengua, que es, hoy, una de las más dinámicas del mundo y la que se halla en mayor ritmo de expansión".

6 El proceso de "profesionalización" de la literatura latinoamericana fue examinada con cierto detalle por Ángel Rama. Según sus argumentos, los escritores del boom se trasladaron a ciudades que contaban con una industria cultural constituida, precisamente, con el fin de insertarse en ciclos productivos profesionales que, en su momento, ya habían sido codiciados sin éxito por los escritores del modernismo. “... [H]a habido ya un grupo de escritores para los cuales la literatura pasó a ser el primer empleo y esto marca de por sí una diferencia notable entre ellos y pone una nota distintiva sobre el fenómeno boom. Lo integraron, principalmente, escritores profesionales" (93). Esta profesionalización, sin embargo y como contrapartida, va a estrechar el lazo que ata la literatura al mercado: “Aunque sigue siendo un hombre con un lápiz y un block de papel; la profesionalización lo suelda de un modo indirecto al mercado, lo que no quiere decir que haga de él meramente un servidor, sino que lo obliga a asumirse como un productor que trabaja dentro de ese marco impuesto. Allí debe operar y triunfar" (94). Este modo de producción emergente para la literatura vernácula va a contrastar con el sistema "tradicional 
estructuras locales, porque evitan la participación de algunos actores y porque la proyección tanto de distribución como de ventas se reduce significativamente, tiende a disminuir el nivel de "profesionalización”. La edición independiente y autogestionada suele concentrar en un solo sujeto o en muy pocos las funciones que en la estructura internacional aparecen encargadas a diferentes especialistas: un editor independiente puede al mismo tiempo ocuparse de la adquisición de contenidos, del diseño, de la impresión, de la distribución, de la promoción y de la venta del libro. Como consecuencia, la escala de producción y puesta en circulación, bajo este formato, van a reducirse sensiblemente.

Pero una producción reducida a nivel local en contextos donde el capitalismo presenta fallas estructurales históricas y profundas adquiere, a su vez, otros matices. Editoriales independientes y autogestionadas existen, sin duda, en todo el mundo, ${ }^{7}$ pero en América Latina, debido a la crisis crónica del capitalismo, la edición artesanal y la autoedición se desarrollan como necesidad $\mathrm{y}$, principalmente, como respuesta al "momento monopólico" de la industria editorial asentada. La precariedad relativa de las condiciones de producción y la progresiva colonización de la industria editorial local de mediana envergadura por parte de los grupos multimedios transnacionales van a alentar la iniciativa independiente que va a llegar a su momento de despegue a comienzos del siglo XXI. Con el acceso a las editoriales tradicionales clausurado, los escritores jóvenes y emergentes interesados por dar a conocer sus escrituras van a volcarse espontáneamente a la edición doméstica. Con escasos o nulos recursos materiales a disposición, los proyectos de publicación van a exigir, a su vez, el desarrollo de técnicas de edición innovadoras y modos de gestión alternativos. Los catálogos en constitución van a incluir, en primer lugar, los textos de quienes impulsan la iniciativa y, en segundo, el de allegados y afines. En cualquier caso, siempre fundados en redes de sociabilización altamente localizadas. Van a surgir la edición a base de cartón y fotocopias y la distribución microbiana en contadas librerías especializadas o directamente orientada al lector en ferias, fiestas y otros eventos culturales.

De este modo, la cadena de agregado de valor se va a comprimir considerablemente así como los costos de producción van a reducirse al mínimo

de las letras”, encarnado de modo ejemplar por José María Arguedas, que Rama va a denominar "aficionado" (94). Mi propuesta -como voy a intentar exponer- sostiene que el modo de producción "artesanal” no solo ha permanecido vigente sino que, desde los años 90 en adelante, incluso se ha expandido a nivel local y que, por consiguiente, debe ser considerado menos "tradicional" que "alternativo".

7 Para el caso de Alemania, por ejemplo, véase Schöllhuber. 
indispensable. Puesto que las "editoriales independientes pequeñas" ${ }^{\text {"8 }}$ suelen publicar escritores nacionales o incluso más locales, no van a existir los costos de traducción presentes en la literatura de circulación internacional. Pero también suele evitarse la mediación de agentes y scouts, con lo que se elude su criterio de selección y sus correspondientes honorarios. El trato se va a establecer directamente entre editor y escritor bajo convenciones de "cooperación" mutua: el autor "cede" contenido y amplía el catálogo a cambio, únicamente, de la publicación, es decir, que los anticipos desaparecen o descienden a sumas puramente simbólicas. Las decisiones de publicación y la línea editorial general van a estar en manos del emprendedor único o del colectivo que impulsa el proyecto, de modo que también desaparecen los gerentes de ventas y con ellos la perspectiva más economicista y costos agregados. Las campañas de promoción van a tener un carácter claramente reducido y las eventuales reseñas van a correr por cuenta de periodistas y otros especialistas comprometidos con la edición independiente o interesados en el libro en cuestión.

Se trata, de acuerdo con las convenciones de la gran industria, sin duda, de una estructura productiva económicamente "ineficiente" con escasos mediadores poco “profesionalizados”. Las pérdidas económicas, por más que exista un esfuerzo por evitarlas, no suelen ser raras. Al contrario, es común no esperar ganancias y, si se dan, apenas sirven para cubrir los costos de materiales e impresión. Los editores o los colectivos editoriales del escenario local, por lo regular, no viven del proyecto sino de otros trabajos más rentables de donde extraen los recursos económicos necesarios para sostener la mínima dinámica productiva en funcionamiento.

Este modo de gestión y producción "artesanal”, por opuesto al "profesional” del escenario internacional, posee, naturalmente, un costo en visibilidad e impacto: se trata de publicaciones que no salen -no están en condiciones de hacerlo- a buscar al gran público, que circulan escasamente por canales alternativos y que se dirigen ante todo a un lector entrenado que selecciona con cierto cuidado el corpus que hace a su biblioteca. Tanto este lector como el editor independiente tienden a fundar su concepto de literatura en variantes del modelo tradicional del valor literario, y la editorial independiente funciona como garantía de calidad para ese lector "exigente". De este modo, a nivel local/nacional, ${ }^{9}$ por el contrapeso que ejerce la edición independiente, se reconstituye el perfil del campo literario tensionado entre fuerzas heterónomas y autónomas: como las condiciones de producción

8 El término "editoriales independientes pequeñas" lo introdujeron tempranamente (2001) Adriana Astutti y Sandra Contreras para distinguir a los emprendimientos editoriales latinoamericanos de baja proyección de ventas de las editoriales con proyección internacional y modos de gestión profesionales.

9 Voy a tratar de establecer ciertas precisiones sobre la alternancia local/nacional en el capítulo IV. 
en la edición independiente suelen definirse por la precariedad económica y de infraestructura y la baja "profesionalización”, el factor dinero al menos en esa zona se desplaza a un segundo o tercer plano. Un libro, en la producción independiente más localizada, se publica, aunque sea en un tiraje irrisorio, porque posee algún tipo de "valor literario". Ese, al menos, suele ser el argumento. Si a posteriori ese mismo libro produce ventas, es un añadido, pero no el factor determinante para la decisión de publicarlo. De modo que, en este nivel de producción, la zona más autónoma del campo logra impregnar con su lógica a la estructura general y organizar las diferentes intervenciones en y sobre el libro en cuestión a partir, hasta cierto punto, del principio del valor literario.

Y la lógica que domina el campo tiene un impacto, aquí también, en la literatura. Como las editoriales independientes no suelen ser rentables económicamente aunque sí, dado el caso, producen un importante rédito en capital simbólico- y los editores no suelen vivir de las ventas de sus publicaciones, como muchos mediadores del campo internacional acá no son necesarios o están completamente ausentes, la publicación no necesariamente tiene que garantizar ganancias $\mathrm{y}$, por lo tanto, amplía el margen de riesgo que puede correr. Es, por lo tanto -en contraposición a la hipótesis que sostiene Pascale Casanova (108 y ss. $)^{10}$-, en el nivel de las literaturas locales, al menos en nuestro presente, donde la experimentación y la disidencia formal hallan su terreno más fértil. El contenidismo, a su vez, se debilita considerablemente porque, como los escritores publicados así como los lectores suelen ser parte de la misma comunidad cultural e interpretativa, la información que podría transmitir la literatura se encuentra en circulación en el contexto inmediato. Al respecto, argumenta Pedro Ángel Palou que "La literatura mundial produce temas, modos reiterados de abordarlos, contenidos universales. La forma estética está fuera de discusión. Sólo desde la periferia [...] puede renovarse profunda, duraderamente" (314). Si la literatura mundial tiende a producir un aplanamiento de las formas estéticas y los contenidos en vistas de responder exitosamente a la demanda internacional que le da sentido, las literaturas locales, particularmente las producidas en condiciones de precariedad económica y de infraestructura, conforman el territorio donde la autonomía literaria (crítica) puede permitirse mayor radicalidad y experimentación.

10 Voy a retomar el punto en el capítulo IV. Quiero dejar anotado, sin embargo, que su método argumentativo para reducir las producciones locales/nacionales a artefactos recurrentes, faltos de valor estético -por oposición a la literatura mundial, cosmopolita-, se funda en una manipulación del objeto: los casos ejemplares que ofrece Casanova se ubican en "Spain during the 1950s and 1960s and Yugoslavia during the 1970s" (111), es decir, en contextos de dictadura o democracia restringida donde los Estados, mediante censura, regulan los parámetros estéticos que van a ser admitidos como expresión nacional. 
También Ana Gallego Cuiñas advierte una diferencia clara en las competencias y atributos que definen al sistema mundial y al local. Escribe:

\begin{abstract}
el primer parámetro de lectura señalado, el "global”, apunta a una literatura que no se asimila totalmente a la representación nacional sino a un objeto "mundial" cuya única marca de identidad es la lengua. [...] "[L]o global" supone un término "neutralizador" que asimila el objeto literario a un modelo dominante más "legible" (vendible), como evidencian los catálogos de las grandes empresas editoriales donde circulan estos textos que se "leen" como "globales". En otro costado, el segundo parámetro de lectura, el "local", se aplica a textos literarios que se "leen" al socaire de tradiciones nacionales y que ponderan el uso de un lenguaje "localista”, “oral”, “ilegible” (“difícil” de consumir). Representan la "subalternidad" y circulan en editoriales independientes -o en "otros" formatos- que fraguan su valor en el capital simbólico dentro del marco de una comunidad de lectores especialistas o "privilegiados".

(“El valor...” 3)
\end{abstract}

Si los estudios académicos concentran su atención únicamente en la literatura que adquiere vida efectiva en el escenario mundial, si se detienen solo en los circuitos de la circulación internacional, se corre el riesgo de que estén reproduciendo irreflexivamente los mecanismos de selección establecidos por la industria editorial internacional. Su corpus no sería exactamente uno organizado de acuerdo con la agenda de investigación, sino uno preseleccionado por los grupos transnacionales de acuerdo con sus naturales intereses. Se estarían ocupando, así, no de las literaturas del mundo y su bibliodiversidad ${ }^{11}$ sino, ante todo, de la literatura seleccionada por la industria editorial para el mundo. Por el contrario, una mirada orientada (también) a

11 Voy a retomar y discutir el término en el capítulo IV. Por el momento, puede considerarse la definición que ofrece la Alianza internacional de editores independientes: "La bibliodiversidad es la diversidad cultural aplicada al mundo del libro. Haciéndose eco del término biodiversidad, se refiere a una necesaria diversidad de las producciones editoriales que se ponen a disposición de los lectores. Si bien los grandes grupos participan, por la importancia cuantitativa de su producción, de una cierta diversidad editorial, eso no alcanza para asegurar la bibliodiversidad, la cual no se mide únicamente por el número de títulos disponibles. Aunque cuiden del equilibrio económico de su editorial, los editores independientes se preocupan ante todo por los contenidos que publican. Sus libros aportan una mirada y una voz distintas, en paralelo a la propuesta editorial más estandarizada de los grandes grupos. La producción editorial de los editores independientes y los canales que privilegian para difundir sus libros (librerías independientes por ejemplo) son por ende imprescindibles para preservar y enriquecer la pluralidad y la difusión de las ideas. Se puede atribuir la invención del término bibliodiversidad a los editores chilenos que crearon el colectivo 'Editores independientes de Chile' a finales de los años 90. La Alianza internacional de editores independientes contribuyó significativamente a la difusión y la promoción del término en varios idiomas, por ejemplo, gracias a las Declaraciones de Dakar (2003), de Guadalajara (2005), de París (2007) y de Ciudad del Cabo (2014). Desde 2010, el Día Internacional de la Bibliodiversidad se festeja cada 21 de septiembre (Día de la Primavera en el Hemisferio Sur)" (Sitio web). 
la producción independiente local, estaría poniendo al descubierto literaturas del mundo que por razones de rentabilidad económica suelen no ingresar en los circuitos internacionales. La operación de una sociología de las ausencias aplicada a la literatura consistiría, por lo tanto, en "mundializar" las escrituras locales; en asignarle visibilidad al corpus que a priori no reúne condiciones para ser asimilado por la gran industria, pero que, sin embargo, puede ser portador de repertorios estéticos, culturales y/o políticos alternativos y disfuncionales, correspondientes, en cualquier caso, a variables locales no hegemónicas. ${ }^{12}$

En las últimas décadas, a medida que se ha ido consolidando el proceso de configuración del actual diseño global y la concomitante concentración editorial que ya ha sido comentada, hemos asistido a un desplazamiento de lo que había sido construido deliberadamente como "literatura latinoamericana" durante los años 60 a un esfuerzo por dejar atrás cualquier postulado identitario e insertar el corpus en gestación en la matriz mayor de la literatura mundial. Se produce, desde entonces, una literatura "latinoamericana" concebida para el mundo sin que la eventual adjetivación posea asociaciones exclusivas o excluyentes. No forzosamente un escritor tiene que mostrar credenciales de pertenencia o residencia, ni su literatura tiene que estar dominada por algún tipo de color local para que pueda ser asociado con la tradición regional o alguna nacional. Sin embargo, -y este es el argumento que me interesa- al mismo tiempo ha cobrado fuerte impulso la producción localizada -la que en efecto tiene lugar en América Latina- que no necesariamente busca "captar" esencias locales, pero que sí se produce bajo condiciones de producción específicas. Habría, de este modo, una "literatura latinoamericana" elaborada por la industria internacional para ser incorporada a la literatura mundial, y, "al otro lado", en la escala local, una literatura latinoamericana producida en condiciones de precariedad material relativa que, no obstante o justamente por ello, favorecen el desarrollo de formas estéticas disidentes y alternativas. En los próximos capítulos, a partir del esquema teórico presentado en estas páginas iniciales, voy a profundizar, por un lado, en algunos mecanismos concretos que desde los años 90 en adelante intervinieron e intervienen en la elaboración de "literatura latinoamericana" o literatura mundial de cuño latinoamericano y, por el otro, en las formas de activismo cultural que impulsan el desarrollo de literatura latinoamericana.

12 En otro lugar (Locane “Más allá...”), introduje la categoría literatura pluriversal en referencia al corpus invisibilizado de literaturas del mundo cuya única marca de identidad sería, precisamente, la heterogeneidad de cosmovisiones y la riqueza formal que puede contener. 